
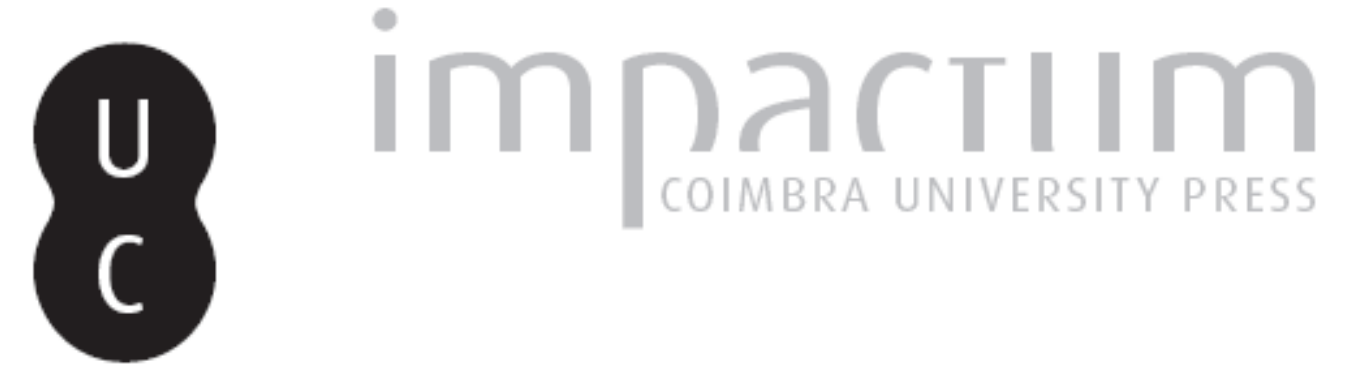

La precipitación y el viento como riesgos climáticos urbanos: el caso de Madrid

Autor(es): $\quad$ Almendros Coca, Miguel Angel; Fernández Garcia, Felipe

Publicado por: Associação Portuguesa de Riscos, Prevenção e Segurança

URL persistente:

URI:http://hdl.handle.net/10316.2/40163

DOI:

DOl:https://doi.org/10.14195/1647-7723_3_4

Accessed : $\quad$ 26-Apr-2023 16:17:55

A navegação consulta e descarregamento dos títulos inseridos nas Bibliotecas Digitais UC Digitalis, UC Pombalina e UC Impactum, pressupõem a aceitação plena e sem reservas dos Termos e Condições de Uso destas Bibliotecas Digitais, disponíveis em https://digitalis.uc.pt/pt-pt/termos.

Conforme exposto nos referidos Termos e Condições de Uso, o descarregamento de títulos de acesso restrito requer uma licença válida de autorização devendo o utilizador aceder ao(s) documento(s) a partir de um endereço de IP da instituição detentora da supramencionada licença.

Ao utilizador é apenas permitido o descarregamento para uso pessoal, pelo que o emprego do(s) título(s) descarregado(s) para outro fim, designadamente comercial, carece de autorização do respetivo autor ou editor da obra.

Na medida em que todas as obras da UC Digitalis se encontram protegidas pelo Código do Direito de Autor e Direitos Conexos e demais legislação aplicável, toda a cópia, parcial ou total, deste documento, nos casos em que é legalmente admitida, deverá conter ou fazer-se acompanhar por este aviso.

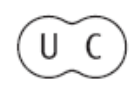




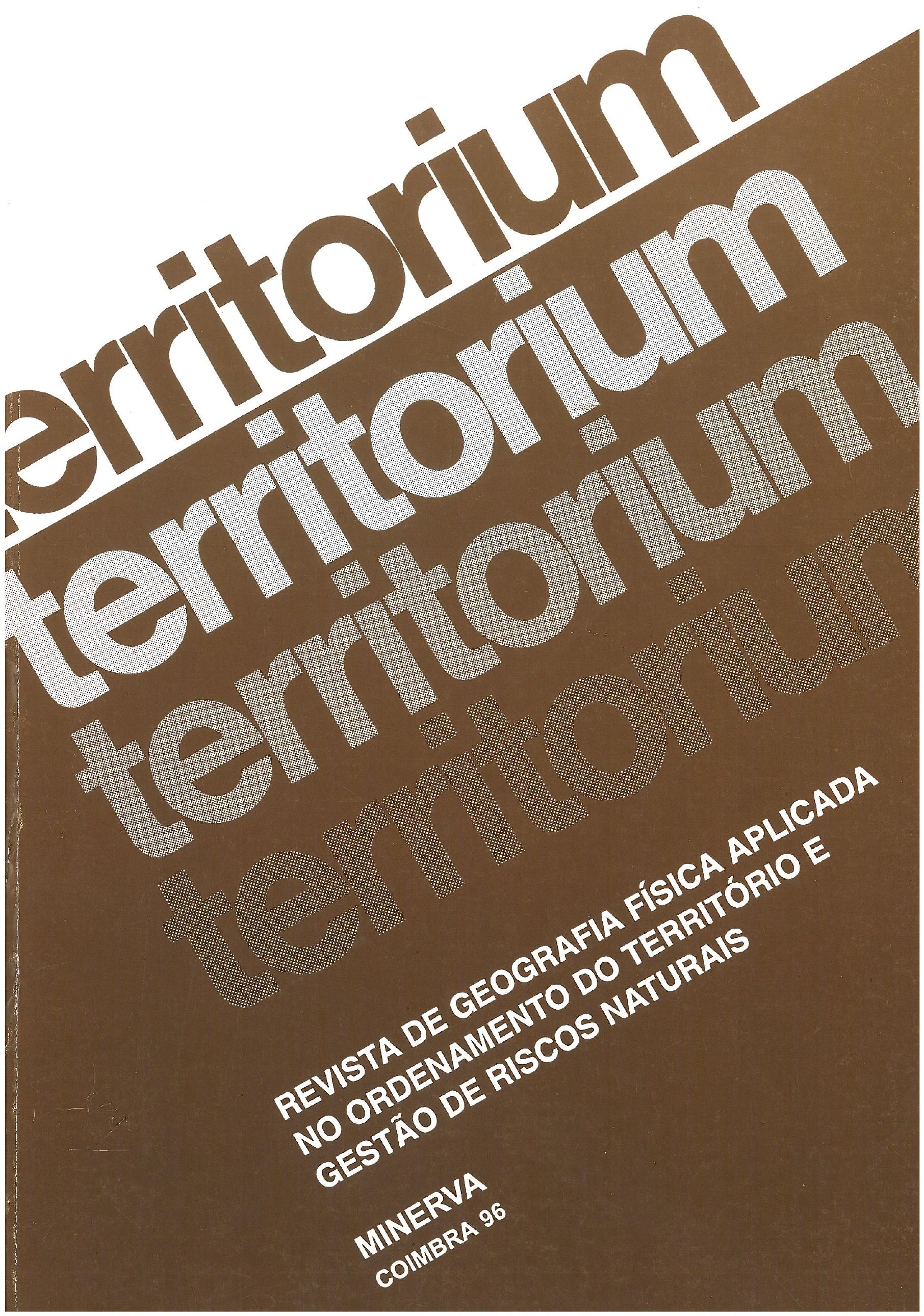




\title{
La precipitación y el viento como riesgos climáticos urbanos: El caso de Madrid.
}

\author{
Miguel Angel Almendros Coca* \\ Felipe Fernández Garcia**
}

\begin{abstract}
Resumo:
A chuva, acima de uma certa intensidade, e o vento, acima de uma certa velocidade, podem considerar-se riscos climáticos. Apresentam-se, neste artigo, os sinistros com eles relacionados na área urbana de Madrid entre 1990 e 1995 e analisa-se pormenorizadamente uma situação concreta de crise provocada por uma tempestade ocorrida em 24 de Junho de 1995.

Palavras chave:

Precipitação, vento, riscos climáticos, Madrid.
\end{abstract}

Résumé:

La pluie, au dessus d'une certaine intensité, et le vent, au dessus d'une certaine velocité, peuvent être considérés des risques climatologiques. Dans cet article, les Auteurs présentent des situations de crise provoquées par les pluies et les vents à Madrid pendant la période de 1990-1995. Ils analysent aussi la crise provoquée par l'orage du 24 juin 1995.

Mots clés:

Précipitation, vent, risques climatologiques, Madrid.

Abstract:

Rain, beyond a certain intensity, and wind, beyond a certain velocity, can be considered climatic risks. In this paper, the Authors present crisis situations in Madrid related with rain and wind in the years 1990-1993

and analyse a case study - the crisis occurred with the storm of the 24 th June 1995.

Key words:

Rain, wind, climatic risks, Madrid.

\section{Introducción}

En un medio artificial como es la ciudad el hombre se siente protegido y de espaldas a las inclemencias del tiempo ya que los riesgos considerados tradicionalmente climáticos están enmascarados por obras de infraestructura realizadas por el hombre. La escasez de precipitaciones es compensada con una red de abastecimiento de agua desde zonas más húmedas (la Sierra), el rayo no preocupa, los vientos fuertes son frenados por el entramado urbano, la niebla y la nieve son menos frecuentes por la isla de calor, el exceso de precipitaciones puede ser corregido mediante la red de alcantarillado, etc.

* Dto. Geografía Física. Universidad Complutense de Madrid.

** Departamento de Geografía. Universidad Autónoma de Madrid.
En este espacio completamente modificado, la protección frente a los riesgos climáticos es aparente, pues la ciudad es como una urna que aisla a los ciudadanos del medio natural y de las inclemencias atmosféricas, pero cuando esta se rompe puede acarrear graves trastornos económicos, incluso los daños pueden ser mayores que en otros lugares. Como señala Ortega (1.991) aparecen involucrados dos subsistemas en interacción, el proceso natural agente y la sociedad afectada o paciente, el grado del riesgo depende de la cuantía del daño y éste en gran medida, depende del nivel de desarrollo tecnológico de la sociedad involucrada.

En el caso concreto de Madrid, que no está sometida a valores climáticos extremos, los riesgos no son espectaculares, ni es previsible que afecten 
gravemente a toda la ciudad de forma directa, pero en cambio un entramado tan complejo va a presentar numerosos pequeños daños de forma puntual e incluso en ocasiones unos daños más importantes pueden repercutir en todo el conjunto urbano, por ello siempre hay que crear y mantener tanto medidas pasivas de defensa y prevención (buena red de alcantarillado), como activas de actuación rápida para corregir los daños producidos (bomberos). Aunque estas inversiones aparentemente no son rentables económicamente, ni desde luego políticamente, puesto que el ciudadano en general no aprecia el beneficio de la prevención, sólo se desarrollan o perfeccionan cuando se han producido los daños.

A modo de ejemplo el 20 de enero de 1.966 los periódicos nos indican que durante $6 \mathrm{~h}$. en Madrid (Retiro) cayeron $34 \mathrm{I} / \mathrm{m} 2$ ocasionando una importante inundación en las zonas deprimidas de la ciudad, como Vallecas donde el agua alcanzó $1 \mathrm{~m}$. de altura y docenas de familias quedaron sin hogar en Orcasitas y Villaverde, los bomberos tuvieron que realizar más de 1.000 salidas urgentes, el alcalde de Madrid indicó que la inauguración próxima del colector Abroñigal evitaría estas inundaciones. Según datos del INM en ese tiempo cayeron $32,8 \mathrm{~mm}$ y en total en ese día 39,7 mm. Así pues Madrid no evitó con la mejora de su alcantarillado el riesgo climático de recibir precipitaciones consideradas muy importantes para esta zona, pero por ejemplo un 15 de junio del 92 cayeron $35 \mathrm{~mm}$., que provocaron 16 salidas por motivos climáticos de los bomberos de Madrid, de las cuales sólo 7 fueron achique de agua, la ciudad se había protegido y los daños fueron muy limitados.

\section{Metodología e fuentes}

Precisamente para evaluar los riesgos climáticos de Madrid vamos a utilizar como fuente, las intervenciones realizadas por los bomberos de esta ciudad. La información de que se dispone y a la que hemos tenido acceso gracias a la amabilidad del Departamento de Extinción de Incendios de Madrid, del que queremos hacer mención especial a $D$. José $M^{a}$ Pérez Soria Jefe de la Subinspección de Plana Mayor y a $D^{a}$. Clara Teruel, asesor técnico de la unidad de relaciones externas, así como del resto de la unidad, sin su colaboración y amabilidad esta información no se hubiese podido utilizar.

De este modo, siguiendo los siniestros a los que asisten los bomberos, podemos conocer dónde, cuándo y de qué tipo son los daños que provoca la climatología adversa de Madrid.

La relación existente entre las crisis atmosféricas y las intervenciones de los bomberos a siniestros de este origen se puede apreciar en el siguiente gráfico (gráf. 1), del 20 de julio del 91, justo a partir de las 17 horas se produce una rápida caída barométrica de $2 \mathrm{mb}$. y una tormenta que deja $7 \mathrm{~mm}$. y se produce una respuesta inmediata con más de 150 llamadas a los bomberos solicitando ayuda.

Los bomberos tienen agrupadas sus intervenciones según tipos, mediante unas claves en total utilizan 179, de ellas seleccionamos, asesorados por el Subinspector Jefe de la Sección de Plana Mayor, 14 claves que están claramente relacionadas con las inclemencias del tiempo, eliminando el resto.

Las 14 claves de intervención están agrupadas en tres apartados que son:

a) daños en los exteriores de las construcciones (saneado de cornisas y aleros, saneado de enfoscados y revocos, retirada de tejas, chapas, saneado de cubiertas y chimeneas, reconocimientos exteriores).

b) daños por agua (achique de viviendas y locales, achique de balsas y charcas, goteras de cubiertas).

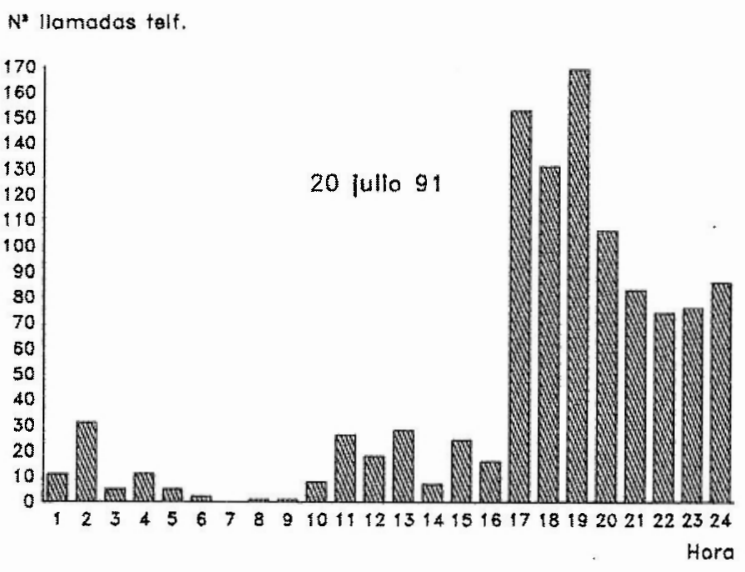


c) incidentes diversos en la vía pública (antenas de TV y radio, carteles y vallas, saneado de árboles).

El análisis se va a circunscribir al periodo 90/93, con un total de 8.168 siniestros por motivos meteorológicos, con un promedio superior a 2.000 intervenciones en siniestros climáticos al año.

\section{Análisis temporal:}

\subsection{El ciclo anual}

Estos tipos de siniestros como puede apreciarse en el graf. 2 y en el cuadro I. siguen un ciclo anual con una menor incidencia y por tanto menor riesgo climático en los meses invernales, cuando predominan las situaciones anticiclónicas, para aumentar durante la primavera coincidiendo con la llegada de situaciones ciclónicas con un aumento de las precipitaciones y del viento, los veranos son irregulares como lo son sus situaciones de baja térmica a la que acompañan fuertes vientos y precipitaciones tormentosos y unos otoños semejantes a la primavera.

Rompen este ciclo y muestran la clara irregularidad temporal de estos riesgos climáticos julio del 91 por las fuertes tormentas de convección y el efecto del viento que se hizo notar especialmente en las vías públicas y sobre todo octubre del 93 que será analizado en detalle.

Cuadro I

Distribución del $\mathbf{n}^{\circ}$ de siniestros por meses y años.

\begin{tabular}{|cccccccc|}
\hline MES & 90 & 91 & 92 & 93 & total & media & Desviación \\
\hline 1 & 114 & 61 & 51 & 57 & 283 & 70.7 & 25.2 \\
2 & 149 & 88 & 66 & 217 & 520 & 130 & 58.7 \\
3 & 133 & 213 & 171 & 137 & 654 & 163 & 32.01 \\
4 & 160 & 142 & 164 & 192 & 658 & 164 & 17.9 \\
5 & 113 & 134 & 216 & 236 & 799 & 199 & 50.6 \\
6 & 125 & 193 & 160 & 278 & 756 & 189 & 56.7 \\
7 & 151 & 552 & 175 & 190 & 1068 & 267 & 165 \\
8 & 173 & 149 & 184 & 269 & 775 & 193 & 45.2 \\
9 & 149 & 133 & 149 & 180 & 611 & 152 & 17 \\
10 & 178 & 138 & 172 & 709 & 1197 & 299 & 237 \\
11 & 137 & 121 & 62 & 148 & 468 & 117 & 33.1 \\
12 & 96 & 64 & 103 & 116 & 379 & 94.7 & 19.1 \\
\hline TOTAL 1.678 & $\mathbf{2 0 8 8}$ & $\mathbf{1 6 7 3}$ & $\mathbf{2 7 2 9}$ & & & \\
AÑ & $(\mathbf{9 0})$ & $(\mathbf{9 1 )}$ & $(\mathbf{9 2 )}$ & $\mathbf{( 9 3 )}$ & & & \\
\hline
\end{tabular}

Por tipo de siniestros los más numerosos son los referidos a daños en el exterior de las construcciones con 4.515 salidas de las que corresponden 1030 (al año 90), 1007 (91),898 (92) y 1580 (93) que responde al mismo ciclo general, aumento en los equinoccios especialmente abril-mayo y octubre-noviembre, un mínimo en diciembre-enero, y una tendencia a disminuir

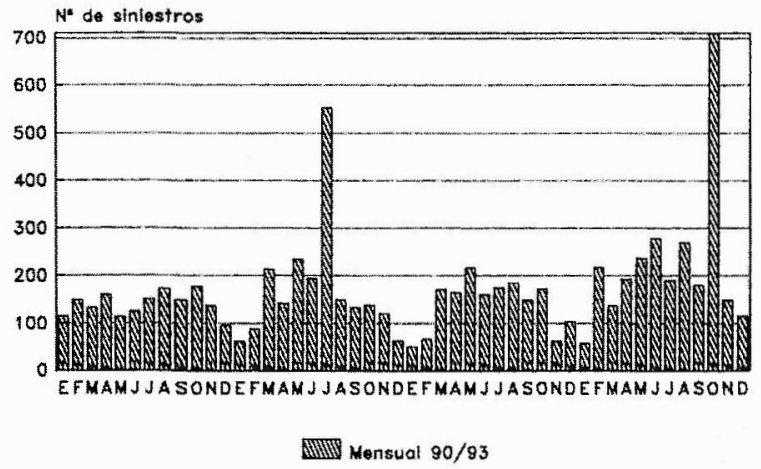

Gráfico 2 - Frecuencia Mensual de los siniestros en Madrid (1990-93)

los daños en verano más marcada que en los otros tipos de siniestros, destaca el año 93 porque en octubre se produjeron 347 intervenciones de este tipo debido a la acción continuada del viento durante casi todo el mes.

En cuanto a los daños por agua, la cantidad de siniestros es sensiblemente menor con 1.067 salidas a siniestros de las que 257 son del 90,273 del 91,221 en el 92 y 316 corresponden al año 93, el reparto general responde a un máximo en los meses veraniegos por las tormentas acompañadas de vientos y precipitaciones convectivas intensas y puntuales, un descenso en Ios equinoccios, para registrar también el mínimo de siniestralidad en invierno.

Los daños en vías públicas con 2586 intervenciones, presentan una notable irregularidad interanual con 391 siniestros en el año 90 y 808, 554, 833 en los siguientes, pero en cambio las variaciones a lo largo de los meses son semejantes a la distribución general de intervenciones en siniestros, por sus características claramente vinculados al viento, el mínimo se registra en los meses invernales, especialmente enero, y el máximo se produce en verano coincidiendo con las tormentas estivales, que superan aunque ligeramente a los siniestros producidos durante los equinoccios, destacando claramente julio del 91 y en menor medida octubre del 93.

\subsection{El ciclo diario}

Tomando las intervenciones diarias se puede comprobar como en general las salidas se producen todos los días, por alguno de estos 14 tipos de siniestros, pero en un número muy escaso siendo frecuentes los valores entre 1 y 3 salidas y las causas muy variadas, puesto que una ciudad del tamaño de ésta con la enorme cantidad de árboles urbanos que 
posee no es extraño que casi todos los días sea necesario sanear alguno o que cualquier antena de TV o cornisa del viejo caserío de Madrid, provoque alguna salida. Pero también se comprueba como durante algunos días muy concretos el número de intervenciones del tipo de las que hemos seleccionado, se incrementa notablemente y pasan a superar las 15 ó 20 , he incluso hay algunas jornadas que han superado las 180 intervenciones, estos casos parecen darse en pulsaciones irregulares que suelen durar 2 días y están vinculados a incrementos importantes en la velocidad del viento y/o en precipitaciones importantes; a veces cuando el fenómeno climático es importante durante dos días se produce un tercero de salidas en menor número para sanear los daños producidos durante la fase previa.

En total se han detectado 33 pulsaciones entre los años 90/92, que corresponden a 58 días.

Son estos umbrales los que hemos seleccionado como indicadores del riesgo climático en nuestra ciudad, entendiendo como un período excepcional durante el cual "se rompe la marcha habitual y cotidiana de la sociedad" (PITA, 1.995).

\section{Los límites del riesgo}

Hemos seleccionado las fechas de estas pulsaciones y los datos recogidos en los partes SYNOP, correspondientes a las variables de viento y precipitación en los observatorios de Barajas y 4 Vientos, que se encuentran situados a unos $20 \mathrm{Km}$ de distancia en los extremos NE y SW de la ciudad, así como del fichero DCAS del observatorio de Madrid-Retiro, en un parque en el centro de la ciudad.

En general se comprueba que es la actuación individual o conjunta de viento y precipitación la responsable de las pulsaciones en los siniestros, con unos límites más o menos claros, a partir de los cuales se dan las condiciones para considerar el día como de riesgo climático.

Estos límites requieren unas precisiones:

a) La actuación del viento. Puede aparecer como única responsable del riesgo, provocando más de 20 salidas por pulsación, en cuyo caso, pasa a ser responsable de riesgo climático en Ia ciudad a partir de 24 nudos, intensidad que es mucho más frecuente en Barajas que en 4 Vientos. Pero también interviene de forma conjunta con las precipitaciones, así vientos de unos 20 nudos pasan a ser responsables de riesgos cuando coinciden con precipitaciones de unos 5-7 $\mathrm{mm} / 3 \mathrm{~h}$. b) Los límites en la precipitación de $20 \mathrm{~mm} /$ día ó $10 \mathrm{~mm}$ en menos de $3 \mathrm{~h}$. son de por sí suficientes para ser responsables de riesgos climáticos. Tenemos también riesgo con vientos de 14-18 cuando coinciden con precipitaciones si estas se dan de forma tormentosa y violenta, como nos indica el tipo de tiempo de la información synop. y'esto aún cuando en los observatorios se han recogido cantidades que no llegan a $1 \mathrm{~mm}$, pero dada la irregularidad en la intensidad y reparto de este tipo de precipitaciones convectivas no podemos cuantificar las cantidades y fuerza del viento que ha provocado la siniestralidad local en la ciudad.

Este apunte de límite de riesgo climático no debe confundirse en el caso de Madrid con peligro de catástrofe, inseguridad en las viviendas o en la vía pública, ya que por ejemplo días con precipitación superior a $20 \mathrm{~mm} /$ día en abril del 91 y 92 no han llegado a provocar en algún ocasión ninguna salida por achique de agua, debido a que las precipitaciones en general no son muy violentas y además la red de alcantarillado se mantiene relativamente limpia por la frecuencia de las precipitaciones en esta época. En cambio precipitaciones menos importantes cuantitativamente, pero producidas en verano y por tormenta, por lo que la caída es más violenta y el tiempo desde las precipitaciones anteriores mayor, con un alcantarillado más obstruido, son suficientes 0,8 $\mathrm{mm} /$ día en Barajas para provocar 12 salidas de achique de agua. Por ello se ha de tener también en cuenta la época del año en que se producen y la situación atmosférica anterior, pasando a ser más importante el riesgo por la irrupción brusca, que por la llegada y desarrollo continuado del fenómeno atmosférico.

En cualquier caso el $n^{\circ}$ de salidas de los bomberos nos indica que el fenómeno afecta a numerosos ciudadanos, pero la intensidad y gravedad, en este tipo de siniestros, es muy limitada.

En cuanto a la rigidez en estos límites de riesgo climático, es preciso tener en cuenta, en primer lugar que se van modificando conforme la ciudad crea las nuevas construcciones, las medidas de seguridad vial mejoran y son menos las viviendas en malas condiciones del casco viejo, por ello, no sería necesario ampliar este trabajo más allá de una serie de 10 años, puesto que la ciudad evoluciona y el resultado puede quedar trastocado, en cambio sí podría ir actualizándose y comprobar cómo los limites se modifican, y en segundo lugar tal y como nos indicaron los profesionales responsables de resolver estos siniestros, el número de salidas en los últimos años no es comparable estadísticamente con los años 60 ó 70 , puesto que en la actualidad la concienciación y vitalidad ciudadana 
es mayor y la utilización de este servicio, se ha incrementado notablemente en cantidad y en cambio se han reducido en intensidad y gravedad, por ello las pulsaciones analizadas en este trabajo, no serían comparables con las de esos años.

A estas primeras precisiones, sobre los límites que provocan las crisis, hay que añadir las siguientes observaciones, sobre el número de siniestros por pulsación y la duración de ésta:

La mayor parte de las pulsaciones se producen a lo largo de dos días. En el primero de ellos se suele producir un gran número de siniestros, coincidiendo con las condiciones atmosféricas que rebasan los límites expuestos y el $n^{\circ}$ es mayor cuanto más altos son los valores de precipitación y viento. Pero en ocasiones no existe correlación entre el evento climático y el efecto, o incluso ambos parámetros son divergentes, como consecuencia del efecto de retardo que se produce en algunos daños. En estos casos se han observado dos tipos de comportamiento:

a) Al reducirse sustancialmente los límites de las variables climáticas en la segunda parte de la pulsación se reducen también los siniestros, aunque por un efecto de debilidad en antenas, cornisas, árboles, etc, no lo hacen con la misma intensidad. Este fenómeno se observa por ejemplo en la pulsación (15/16 abril 91) cuando el primer día se producen 14 salidas por efecto de fuertes precipitaciones con unos $20 \mathrm{~mm} /$ día y cerca de $10 \mathrm{~mm}$ en $3 \mathrm{~h}$., en cambio el segundo día aún se producen 12 salidas aunque deja de precipitar y el fuerza del viento (9 nudos) no puede explicar que se mantenga la pulsación.

b) Por el contrario si el fenómeno climático se mantiene semejante o ligeramente más intenso el segundo día, las salidas se incrementan notablemente, como ocurrió en la pulsación 7 en julio del 91 cuando unas precipitaciones tormentosas, por baja térmica por la tarde provocan 62 salidas a siniestros, al día siguiente unas tormentas con valores semejantes nos elevan las salidas a 184 y aunque los dos días siguientes no precipita y el viento no supera los 10 nudos, los daños provocados se alargan y provocan 97 salidas en el tercer día y aún se producen 26 en el cuarto.

Los ejemplos expuestos indican la dificultad de establecer una relación causa-efecto entre las variables climáticas y la intensidad de los daños provocados y, sobre todo, mostrar cuantitativamente dicha correlación.

Debido a lo significativo que fue octubre del 93 , en el que prácticamente todo el mes fue de alta siniestralidad, se pretendió realizar un estudio estadístico con la información diaria de Barajas de la hoja climática, de ella seleccionamos 6 variables independientes:

Racha máxima de viento.

Dirección del viento en grados.

Recorrido del viento en $\mathrm{km}$ de las 00 a las $24 \mathrm{~h}$.

Precipitación en los 4 grupos horarios de medición.

Intensidad máxima de precipitación en $\mathrm{mm} / \mathrm{h}$.

Precipitación en $24 \mathrm{~h}$.

El trabajo estadístico se realizó con el programa statgrahpics

y por el método Stepwise.

Las 6 variables conjuntamente no presentan suficiente fiabilidad respecto a los daños producidos en los siniestros.

Si eliminamos las variables no ajustadas (Backward), con un F-remove de 4, los resultados son los siguientes:

$\begin{array}{llccc}\text { Siniestros ........... Variable indep. } & \text { Nivel sig. } & \mathrm{r}^{2} & \text { Coef. Correl. } \\ \text { Construcción ....... Km/24h } & 98 \% & .44 & 0.34 \\ & \text { Precip/día } & 96 \% & & \\ \text { Agua ................ Precip/4 } & 100 \% & .71 & 0.69 \\ \text { Arboles ............. Km/24h } & 100 \% & .54 & 0.69 \\ \text { Vía pública ......... Km/24h } & 100 \% & .52 & 0.49 \\ & & & \\ \text { Total ................. Km/24 h } & 100 \% & .53 & 0.45 \\ & \text { Precip/dia } & 95 \% & & \end{array}$

Que nos indica como todas las variables de daños en la ciudad son explicados al menos por una de las independientes y que estas son fundamentalmente el recorrido del viento en $\mathrm{Km}$ en 24 horas y las precipitaciones caídas en esas 24 h. Las correlaciones más fuertes son por supuesto la de aquellos daños relacionados más claramente con un fenómeno concreto tal y como ocurre con el agua y su relación con la precipitación caída en uno de los 4 grupos horarios y los daños en los árboles relacionados con el recorrido del viento. Los daños producidos en el exterior de las construcciones y en el $n^{\circ}$ total de siniestros presentan unos resultados menos claros, pero también las causas son más complejas y su relación con el segundo día o siguientes, bien para favorecer el aumento de los daños en unos casos o su descenso pero con retraso, en otros desvían los resultados estadísticos, por ello la relación entre estas variables se puede apreciar mejor en gráficos.

En el gráfico 3 se comparan las lluvias con el número de salidas o siniestros relacionados con los daños por agua. Se observa una clara correlación entre ambos y el intervalo del 9 al 11 es un buen ejemplo de como los efectos negativos se van acumulando cuando el evento climático dura varios días. 


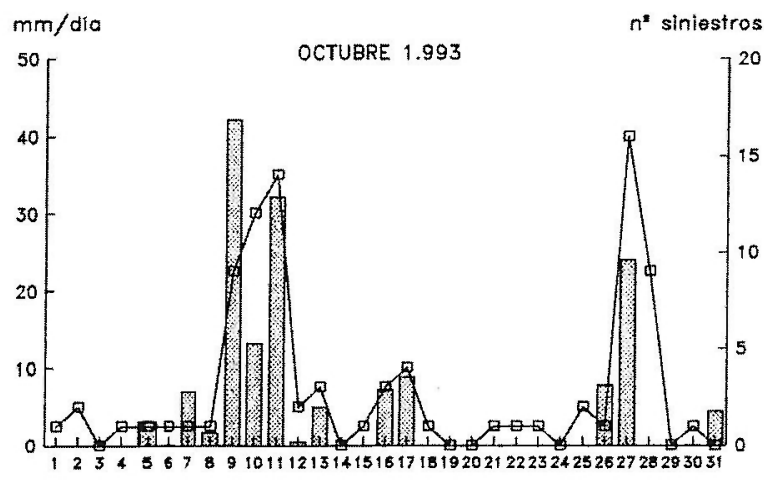

D DAROS AGUA PRECIPITACION

Gráfico 3

En el gráfico 4, se observa la correspondencia entre la velocidad del viento y el número de siniestros, pero también como después de unos días con fuerte y reiterada acción del viento la ciudad se ha ajustado y desciende sensiblemente el $n^{\circ}$ de siniestros como ocurre después de los 3 primeros días de acción del viento, tras los cuales una ligera disminución de la acción de éste, se traduce en un fuerte descenso de la siniestralidad y en cambio valores semejantes como ocurre los días 22 y 23 pero después de un periodo más calmado provoca en cambio un claro incremento en la siniestralidad.

En el gráfico 5 por último, se representa la acción conjunta de las variables precipitación y viento que aclaran mejor el incremento de siniestralidad de los días $9,10,11$ y del $26,27$.

\section{El clima y los riesgos}

El proceso establecido hasta aquí ha consistido en conocer las pulsaciones en los siniestros y conocidas

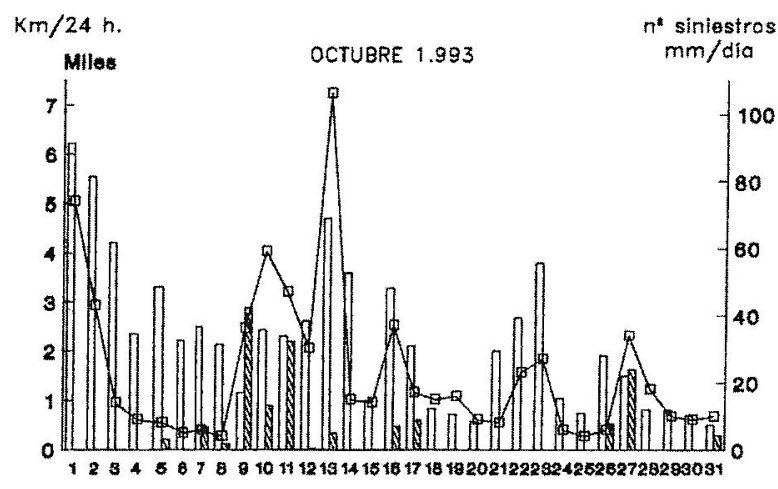

$\rightarrow$ TOTAL DANOS PRECIPITACION $\square$ VIENTO

Gráfico 5 diversas variables climáticas y sus intensidades establecer los límites a partir de los cuales se ha producido la pulsación, es decir conocidas las consecuencias, buscar las causas y establecer el límite del riesgo.

Pero quedaba en el aire el interrogante de plantear la metodología inversa, al analizar las causas aisladamente, deberían de coincidir con los efectos encontrados. De no cumplirse esta hipótesis los límites de riesgo no serían válidos.

Para ello hemos buscado los días que llegan o superan los valores arriba indicados con la idea de comprobar qué porcentaje corresponden a pulsaciones y estudiar cuándo y porqué no han producido un aumento claro de siniestralidad.

Climáticamente como ya hemos indicado anteriormente la ciudad de Madrid no presenta valores extremos importantes:

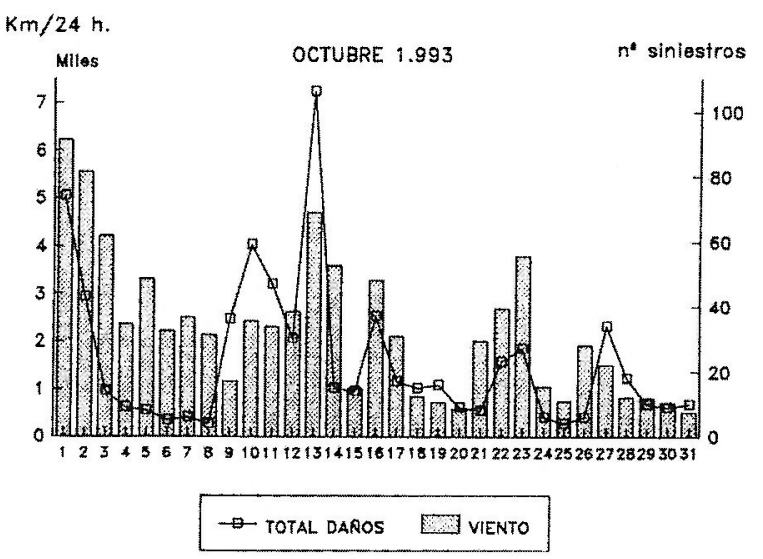

Gráfico 4

Según datos del Atlas Geográfico Nacional. Climatología. MOPTMA. y de los Valores normales y estadísticos de estaciones principales (1.961-1.990), para el observatorio Meteorológico de Madrid. Retiro, INM. Id. de Barajas.

a) En Precipitación el riesgo de tormentas es de unos 10 días en verano, 5 en primavera, 4 en otoño y 0 en invierno y los meses con más días de tormentas en Retiro (2) son junio $(3,2)$ y julio $(2,7)$, y en Barajas (3) son junio $(3,7)$ y mayo $(2,8)$, aunque en algunos años concretos se ha llegado a 10 días en un sólo mes.

Las precipitaciones máximas en un día fueron de $87 \mathrm{~mm}$ en septiembre del 72, pero son habituales ser de $20 \mathrm{a} 40 \mathrm{~mm} /$ día incluso algunos años no se han llegado a estos valores ningún día. 
Siguiendo los datos synop. de Barajas y 4 Vientos del $90 / 93$ comprobamos que existe una gran irregularidad interanual en los valores extremos de la precipitación, por ejemplo:

En 1.990 se registraron 3 días con precipitación superior a $20 \mathrm{~mm} /$ día y los tres casos se corresponden con pulsaciones

En el 91 fueron 4 los días con precipitación $>$ de $20 \mathrm{~mm} /$ día y tres de ellas forman parte de pulsaciones encontradas el único caso en que esto no ocurre sí esta vinculado a una pulsación, pues fue el 9 de octubre con $20,6 \mathrm{~mm} /$ día producidos en la tarde y aumentaron hasta superar en ambos los $21 \mathrm{~mm}$ al día siguiente. En cambio el efecto en la siniestralidad se aprecia a partir del día 10 y esta pulsación completa duró 4 días (la más extensa de las estudiadas), así pues es un sólo caso en el que se produjeron este año precipitaciones importantes y no se reflejo en la siniestralidad.

En el 92 fueron 7 días de los cuales 5 formaron pulsaciones, los dos restantes corresponden a un día tormentoso de julio que no produjo precipitaciones importantes en Cuatro Vientos y el 11 de octubre con $24 \mathrm{~mm}$ de lluvia débil continua.

Asíen general el riesgo de que $20 \mathrm{~mm} /$ día provoquen una crisis de siniestralidad es del orden del $80 \%$ en Barajas y del $90 \%$ en 4 Vientos.

La intensidad es menos clara pues precipitaciones superiores a $7 \mathrm{~mm}$ en 3 horas en Barajas, en el trienio 90/92 se dieron en 38 días, de los cuales 18 corresponden a pulsaciones que además coinciden aproximadamente con precipitaciones totales diarias de unos $20 \mathrm{~mm}$., nos da un riesgo del $47,4 \%$, pero de los 20 días restantes sólocuatro no corresponden a precipitaciones puntuales tormentosas.

b) El viento. Las rachas máximas de viento en los últimos 30 años han sido $111 \mathrm{Km} / \mathrm{h}$ en Retiro, 122 en Barajas y 124 en 4 Vientos, pero los valores máximos mensuales habituales son de 50 a $60 \mathrm{Km} / \mathrm{h}$. y los anuales de poco más de $80 \mathrm{Km} / \mathrm{h}$.

De acuerdo con los datos synop en el trienio de análisis, en Barajas de los 18 días con viento superior a 24 nudos, 11 corresponden a pulsaciones, poco más del $61 \%$. Y en Cuatro Vientos el porcentaje es semejante.

En cuanto a los días con más de 20 nudos y precipitación superior a $4 \mathrm{~mm} /$ día coincide en un $90 \%$.
Así pues en líneas generales podemos afirmar que los límites establecidos anteriormente, sí son fiables.

\section{Distribuición espacial}

La distribución por distritos de las intervenciones del cuerpo de bomberos, es otro aspecto significativo del riesgo climático urbano y del grado de siniestrabilidad por el que podemos conocer los espacios urbanos más conflictivos y los más seguros, aunque indudablemente la siniestrabilidad depende fundamentalmente del grado de deterioro de los distritos afectados.

El número de siniestros en cifras absolutas, no nos permite comparar la siniestralidad de unos distritos con otros, puesto que por ejemplo la extensión de los mismos y el grado de utilización del suelo es muy diferente como Fuencarral que incluye el monte del Pardo y tiene una enorme extensión no edificada y lo mismo ocurre con otros distritos periféricos, donde además predomina el erial, por ello para esta aproximación hemos utilizado el número de habitantes para poder comparar los diferentes distritos, estableciendo la siniestralidad por cada $1.000 \mathrm{hb}$, como podemos apreciar en el cuadro II.

El distrito centro (3.27) es con diferencia el más conflictivo, registrando más del doble de siniestralidad respecto a los siguientes distritos, que son los Ensanches, destacando Salamanca y Chamberí, en los que se supera 1.2 siniestros por cada $1.000 \mathrm{hb}$ y año, las causas podrían ser:

a) Concentración de edificación e instalaciones antiguas, especialmente en el casco viejo (Centro). En menor medida los Ensanches con un caserío en general también antiguo y con alta densidad, pero intercalado con edifícación moderna.

b) Alto índice de densidad poblacional 302-336$391 \mathrm{hb} / \mathrm{Ha}$. respectivamente.

c) La centralidad favorece una mayor densidad peatonal, comercial, lugares de ocio, etc.

Los distritos periféricos con un mayor porcentaje de espacios abiertos, no edificados, caserío con menor antigüedad y menor densidad de habitantes contiene los menores valores de riesgo, sin llegar a 0.3 siniestros por cada $1.000 \mathrm{hb}$. y año, se encuentran Villaverde, Fuencarral, otros distritos con una siniestralidad 10 veces menor que el Centro son Villa Vallecas y Hortaleza.

Por tipo de siniestros las resultados son semejantes con las siguientes matizaciones: 
CUADRO II.

Siniestros registrados durante el periodo 90/93, expresados en relación a la población de cada distrito (por cada 1.000 hab/año).

\begin{tabular}{|lccccc|}
\hline DISTRITO & CONST. & AGUA & VIA P. & TOTAL & POBLACIóN ${ }^{*}$ \\
\hline 1.- CENTRO & 2.48 & 0.31 & 0.48 & 3.27 & 136.972 \\
2.- ARGANZUELA & 0.41 & 0.09 & 0.36 & 0.87 & 116.960 \\
3.- RETIRO & 0.35 & 0.06 & 0.23 & 0.64 & 127.709 \\
4.- SALAMANCA & 0.81 & 0.15 & 0.39 & 1.35 & 156.657 \\
5.- CHAMARTIN & 0.37 & 0.10 & 0.27 & 0.74 & 146.214 \\
6.- TETUAN & 0.47 & 0.10 & 0.23 & 0.80 & 147.425 \\
7.- CHAMBERI & 0.75 & 0.10 & 0.35 & 1.20 & 161.167 \\
8.- FUENCARRAL & 0.08 & 0.06 & 0.13 & 0.27 & 207.504 \\
9.- MONCLOA & 0.37 & 0.14 & 0.38 & 0.88 & 105.826 \\
10.- LATINA & 0.15 & 0.06 & 0.13 & 0.34 & 271.625 \\
11.- CARABANCHEL & 0.18 & 0.09 & 0.12 & 0.38 & 234.215 \\
12.- USERAS & 0.19 & 0.07 & 0.13 & 0.39 & 120.664 \\
13.- PTE VALLLECAS & 0.22 & 0.06 & 0.15 & 0.44 & 225.283 \\
14.- MORATAZ & 0.11 & 0.06 & 0.11 & 0.28 & 105.711 \\
15.- C. LINEAL & 0.15 & 0.04 & 0.20 & 0.39 & 225.960 \\
16.- HORTALEZA & 0.10 & 0.05 & 0.18 & 0.33 & 138.789 \\
17.- VILLAVERDE & 0.11 & 0.08 & 0.11 & 0.29 & 124.941 \\
18.- VILLA VALLECAS & 0.12 & 0.06 & 0.12 & 0.30 & 59.856 \\
19.- VICALVARO & 0.28 & 0.04 & 0.17 & 0.48 & 40.576 \\
20.- SAN BLAS & 0.16 & 0.07 & 0.17 & 0.39 & 128.762 \\
21.- BARAJAS & 0.15 & 0.05 & 0.21 & 0.41 & 34.597 \\
\hline TOTAL & 0.37 & 0.09 & 0.21 & 0.68 & 3.017 .423 \\
\hline
\end{tabular}

En cuanto a los daños en exterior de construcción aunque los resultados son iguales a los totales, la diferencia entre el Centro con el caserío más antiguo y degradado y el resto de distritos se acentúa porcentualmente llegando casi a $2500 \%$, mayor el riesgo que en las zonas periféricas de Villaverde u Hortaleza, eincluso un $300 \%$ mayor que en Salamanca, el segundo distrito más conflictivo.

En los daños por agua el Centro de nuevo marca el máximo, pero en este tipo de siniestros las diferencias se reducen, aunque sigue siendo la periferia como Vicálvaro u Hortaleza las que registran los mínimos.

Por úl timo los daños en la vía pública aunque de nuevo son más importantes en el Centro, las diferencias con los distritos de Ensanche son mínimos, destacando entre éstos Moncloa donde encontramos grandes espacios arbolados (Ciudad Universitaria, Parque Oeste, Dehesa de la Villa o la Casa de Campo) y el saneado de árboles es precisamente el tipo de siniestro en vía pública más frecuente, en el extremo opuesto se encuentran Moratalaz y Villaverde.

\section{Análises de una situación concreta}

Analizamos en detalle una pulsación reciente y fuera del grupo general de 4 años (90/93) de estudio. La tormenta estival de la tarde del 24 de junio de 1.995 , que causó graves trastornos al conjunto urbano y un gran número de intervenciones de los bomberos.
Las condiciones atmosféricas como se pueden apreciar en el boletín Meteorológico diario, en la última decena de junio del 95 nos presenta una situación de altura con el Jet Stream ondulante con un potente anticiclón sobre las Islas Británicas que en superficie es mayor de $1032 \mathrm{mb}$, con un circulación meridiana porCentro Europa que nos deja una profunda vaguada sobre el norte de Italia y una bolsa de aire ciclónico aunque no demasiado potente, pero casi extrangulada sobre la Península Ibérica con una advección de aire frío en altura del NE, que en los días sucesivos, ya separada del Jet derivará lentamente hacia el SW Peninsular para pasar a formar una situación difluyente y finalmente el día 30 de junio la baja de desplaza hacia el NW peninsular y con ello además de continuar debilitando el anticiclón Céltico pasa el Jet a una circulación en "omega". Mientras que en superficie se encontraba una depresión térmica sobre el norte de Africa que se mantuvo invariable y favoreció la inestabilidad superficial.

El resultado fue que en los días previos a esta gota fría 21/23 la situación era de inestabilidad por la tarde con formación de nubes de evolución (cumulonimbos) que a ultimas horas de la tarde provocaban pequeñas tormentas que en Madrid en ningún observatorio superó los $0,5 \mathrm{~mm}$. Pero el día 24 con la llegada de la vaguada en altura se produjeron tormentas generalizadas por toda España peninsular, que en algunos casos fueron localmente importantes 
en Madrid y en Castilla-La Mancha, situación que se mantiene en los días siguientes, pero desplazándose las tormentas más importantes hacia Andalucía y el Estrecho (Melilla el 25-26 de junio recoge $133 \mathrm{~mm}$ ), cuando la situación se encuentra reforzada por una baja en superficie.

Así pues en Madrid el día 24 poco antes de las 21 h. se inicia una importante tormenta al encontrarse la masa de aire convectivo (unos $30^{\circ} \mathrm{C}$ en superficie) que ascendía con una situación depresiva en altura con embolsamiento de aire frío $\left(-12{ }^{\circ} \mathrm{C}\right.$ a $5700 \mathrm{~m}$.). La precipitación fue desigual en su reparto: En el centro de la ciudad, Madrid-Retiro, la precipitación no llegó a los $16 \mathrm{~mm}$, aunque su intensidad fue de 33 $\mathrm{mm} / \mathrm{h}$; ; en el NE de la ciudad Madrid-Barajas cayeron $21 \mathrm{l} / \mathrm{m} 2$, con una intensidad de $45,6 \mathrm{~mm} / \mathrm{h}$; aun más importante fue la tormenta sobre el NW en MadridCiudad Universitaria con $55 \mathrm{~mm}$; y el mayor registro se produjo en el SW de la ciudad sobre Madrid-4 Vientos con 79,4 mm y una intensidad de $52,2 \mathrm{~mm} /$ h. Mientras que el viento llegaba a los $60 \mathrm{Km} / \mathrm{h}$ en Barajas.

La respuesta en las salidas de los bomberos al inicio de la tormenta es casi inmediata a las 20:59 hora oficial ( $2 \mathrm{~h}$. menos solar), se produce la primera intervención por la tormenta, una balsa de agua en la vía pública en el extrarradio E de Madrid. Durante la primera hora de tormenta sólo se producen 4 intervenciones en el E y NE de la ciudad, ya en la hora siguiente son las 8 intervenciones y entre las 23 y 24 h. coincidiendo con la intensidad máxima se llega a 11 intervenciones, y este índice de siniestralidad de unas 10 intervenciones hora se mantiene hasta las 5 de la mañana, pero la cola de la pulsación se arrastrará todo el día 25 incluida la noche hasta el día 26. En total se habían recibido 1.600 Ilamadas de teléfono a los bomberos y se habían acudido a más de 130 siniestros de origen climático en 36 horas, desde las 21 h. del 24 a las 9 de la mañana del 26.

Las áreas más afectadas habían sido durante la tormenta la zona NW de la ciudad, así como el N, destacando las numerosas inundaciones de la vía pública, y de las conexiones de carreteras de entrada a Madrid en sus confluencias con la M-30, atrapando numerosos vehículos incluido un autobús urbano. Sólo en la carretera del Pardo con la M-30 quedan 15 coches con ocupantes y la inundación de la estación de Metro de Parque de las Avenidas, atrapa a 60 personas en el NE de Madrid.

Después de la media noche, durante el resto de la noche las intervenciones más importantes se producen en la Casa de Campo en el W y en especial en el eje del río Manzanares que a la altura de la Casa de Campo, se desbordó hacia las 00:10 h., inundando la M-30, se recatan en lancha numerosas personas atrapadas y quedan bajo las aguas decenas de coches bajo el puente del Rey y el de Segovia y abren las compuertas $n^{\circ} 9$ y 10 . Hacia las 3 h. se inunda la línea de Metro 4 entre las estaciones de Alfonso XIII y Avda. de la Paz, también en el NE de la ciudad.

Y a las 3:10 h. fruto de las intensas precipitaciones que habían sido más importantes en el $\mathrm{W}$ de la ciudad los secos arroyos de la Casa de Campo presentan unas riadas hacia el Manzanares que provocarán inundaciones en la Carretera de la Coruña y en la M-30 que circulan junto al río, especial virulencia tendrá la producida hacia el final del arroyo Meaques que coincide aproximadamente con el lago de la Casa de Campo cuyo muro de protección cedió provocando una avalancha sobre el Manzanares, que al encontrarse con un espacio deprimido en la M-30 bajo los puentes del Rey y Segovia inundó por segunda vez esta misma área, formando una balsa de $3 \mathrm{~m}$ de profundidad, con 80 coches atrapados.

Ya en la mañana del día siguiente aún continúan siendo muy numerosas las intervenciones en inundación de sótanos, terrazas, etc. Y comienzan a tener importancia los movimientos de tierras con caída de muros, rotura de colectores, y ya al final del día y en la noche del 25 al 26 sin dejar de acudir a inundaciones pasan a tener importancia los daños menores en construcción, en andamios y en edificios y viviendas en obras.

Así pues concluyendo los daños más importantes se producen durante la tormenta y poco después, las áreas más afectadas son las zonas periféricas en un arco desde el W al E, pasando por el $\mathrm{N}$ y las menos afectadas las centrales y sur, donde se habían producido menores precipitaciones.

Analizando los mayores daños se pueden establecer las causas y con ellos las medidas a tomar para evitar la reiteración de un tipo de daños. Y estos se habían producido:

a) En los espacios sin canalizaciones importantes donde el agua de lluvia puede discurrir superficialmente sobre antiguos arroyos, en los que además por la urbanización se colapsan y taponan los recorridos naturales y sus desagües. Desgraciadamente se produjo un fatal accidente y falleció una mujer que pernoctaba en una obras de Aravaca cuando el agua la arrastró a través de un aliviadero del arroyo Meaques.

b) Sobre las modernas vías de circulación rápida en general y en especial como puntos negros habituales 
encontramos las confluencias de vías de este tipo que suelen coincidir con espacios deprimidos para realizar los cruces, en las que frecuentemente no encuentra salida natural en agua y los medios artificiales para compensar este problema se encontraban atascados por falta de limpieza o saturados por la fuerte intensidad de las lluvias en cualquiera de los casos no existía una señalización en los muros para localizar los desagües y sumideros, lo que dificultó gravemente la solución del problema y a ello hay que añadir la obturación de las medianas de los desdobles de las calzadas, que en muchos casos actuaron como tapón.

Además de localizar los puntos negros y resolver las causas reales que provocan los daños y que no es la climatología adversa, puesto que esta sólo es el desencadenante, sería preciso tal y como indica el Departamento de Extinción de Incendios en un informe en el que pide una mayor coordinación entre los Jefes de Guardia de la Policía Municipal y Bomberos, y en situaciones semejantes un contacto permanente $y$ eficaz con el Instituto Nacional de Meteorología, y los servicios de mantenimiento de las compuertas del Manzanares; así como la adopción de un plan de alerta que garantice un sistema de cortes de tráfico en puntos conflictivos que impida la retención y colapso de vehículos por embalsamiento de agua.

\section{Consideraciones finales}

Una fuente interesante para conocer los riesgos climáticos en las ciudades podría ser el registro de salidas de los bomberos, tal y como hemos analizado en Madrid.

En ellas además del número de intervenciones, se especifica el tipo de siniestros, que aparecen agrupados en: daños en el exterior de construcciones, por agua de lluvia y daños en la vía pública

Se han tenido en cuenta todas las salidas de los bomberos de Madrid a siniestros por motivación climática directa o indirecta (deterioro previo o vejez). Y sólo se han considerado periodos de riesgo o pulsaciones, aquellos días en los que las salidas superaban los 15 siniestros.

Hemos podido comprobar como la totalidad de las pulsaciones coinciden con condiciones atmosféricas de precipitación superior a $20 \mathrm{~mm} /$ día o $10 \mathrm{~mm}$ en menos de 3 horas; de viento superior a 24 nudos (unos $44 \mathrm{Km} / \mathrm{h}$ ); o de la acción conjunta de ambos elementos climáticos en cuyo caso Ios límites para provocar una pulsación se pueden reducir sensiblemente especialmente si coincide con tormentas convectivas.

Estos periodos de aumento de la siniestralidad por motivos atmosféricos se caracterizan por la irregularidad, aunque se han podido establecer una serie de rasgos generales característicos como son: una tendencia a la concentración en periodos de dos días; mayor frecuencia en verano con situaciones convectivas y tras periodos largos de sequía que reducen la capacidad de absorción del alcantarillado y acentúan la siniestralidad, y una drástica reducción durante los inviernos con situaciones anticiclónicas.

Las zonas más castigadas son las del centro con un caserío más degradado y de los ensanches. Las áreas menos afectadas son las periféricas con un caserío más moderno y más espacios abiertos.

En la actualidad se están analizando pormenorizadamente cada uno de los partes del Departamento de Extinción de Incendios de Madrid, conociendo los lugares concretos del siniestro los detalles e incidencias de éste, la hora exacta en que se produce, etc., para realizar un mapa de distribución del riesgo según tipo de inclemencia y siniestro, así como la concentración o distribución horaria de los siniestros en función de las condiciones atmosféricas de esos tramos horarios, este trabajo es continuación del primer avance presentado por ambos en la II Reunión del grupo de climatología de la AGE. sobre el tema de situaciones de riesgo climático en España celebrado en Jaca en 1.995.

\section{BIBLIOGRAFÍA.}

ALMENDROSCOCA, M. A. y FERNÁNDEZGARCÍA, F. (1995) "Riesgos de origen climático en la ciudad: El caso de Madrid". Situaciones de riesgo climático en España. Instituto Pirenaico de Ecología. Jaca.

ORTEGA ALBA, F. (1991): "Incertidumbre y riesgo natural". Sociedad y Territorio, Valencia. Aso. de Geógrafos españoles 99-108.

PITA, F. y AGUILAR, M. (1995): "Riesgos naturales" y "Los riesgos climáticos en España". Curso sobre impactos y riesgos climáticos. Madrid. CEDEX. 